Annals of Plant Sciences

ISSN: 2287-688X

OPEN ACCESS

www.annalsofplantsciences.com

Research Article

\title{
Extraction, characterization \& applications of natural dyes
}

Bushra Khan $^{1 *}$, Ramya Sindhyan ${ }^{1}$, Anushi Divan ${ }^{2}$, Sejal Rathod ${ }^{3}$

${ }^{1}$ Dept of Biotechnology, K.C College, Mumbai, Maharashtra -400001, India.

2Jai Hind College, Churchgate, Mumbai, Maharashtra 400020, India

${ }^{3}$ Kishinchand Chellaram College, Churchgate, Mumbai, Maharashtra 400020, India.

Received: 14-08-2018; Revised: 12-09-2018; Accepted: 23-10-2018

\begin{abstract}
Artificial dyes, widely used in everyday items, come with side effects not known to the common man. They are toxic, harmful \& mostly carcinogenic. There has been a worldwide increase in the demand of natural dyes due to its therapeutic properties \& no known side effects. The current research aimed at extracting dyes of different colors from natural plant sources, carrying out its characteristic analysis \& its applications. The dyes were tested for their antimicrobial ability and were found to be inhibitory to common organisms like S. typhi, C. diphtheriae and S. aureus, etc. Phytochemical tests were performed and they confirmed presence of several important metabolites like Phenols, Tannins, Terpenoids, and many more. The dyes were tested for anti-oxidant activity using the FRAP assay and antioxidant levels ranging from 0.62 to 2.00 were obtained. Preparations of cosmetics like lip balms, was carried out, and dyes were also tested as natural food coloring agents. The results indicated that the dyes caused inhibition of wide range of bacteria and proved to be good sources of anti-oxidants. They departed a natural color to food items and hence can replace artificial food colorings. Thus the use of natural dyes should be promoted, as they are cheap, biodegradable and feasible.
\end{abstract}

Keywords: Natural Dyes; Antimicrobial; Phytochemicals; Anti-Oxidant; Cosmetics; Food Coloring

\section{Introduction}

Dye is a natural or synthetic substance used to impart color to, or change the color, of textiles, paper, leather, and other materials, such that the coloring is not readily altered. (1) Artificial dyes have been used widely since its inception. They are used in everyday items like clothes, food products, cosmetics, etc. The harmful effects of these dyes, however, go unnoticed by the people. In recent years, due to the scientific development, the adverse effects of these dyes are being discovered, and they have been found to cause or contribute to various allergic reactions, toxicity, mental disorders like ADHD, tumor formations, etc. Recent studies have shown that many commonly used artificial dyes contribute to cancer, including brain and testicular tumors, colon cancer, and mutations. (2) These dyes not only pose a risk to the biotic components, but also are responsible for degradation of abiotic components like soil \& water. The contamination of soils and waters by dye-containing effluents is of great environmental concern. ${ }^{(3)}$

Dyes obtained from natural materials like plant leaves, roots, bark, insects, etc were the only dyes used for the coloring of textiles \& other products, until the discovery of the first synthetic dye in 1856. Rapid research in synthetic chemistry, supported by industrialization led to the development of synthetic alternatives, which gradually pushed the natural dyes into a stupor. However, recent trends of irrational use of artificial dyes and numerous environmental and health issues have once again revived interest in

\section{${ }^{*}$ Corresponding Author:}

Ms. Bushra Khan,

Dept of Biotechnology, K.C College,

Mumbai, Maharashtra -400001, India.

E-mail: bushrak34@gmail.com production \& use of natural dyes. Natural dyes are eco-friendly since they are renewable and biodegradable.(4) Natural dyes are harmonizing \& soothing to the human eye. Some of its constituents are anti-allergens, safe for skin contact and are mostly non-hazardous to human health. ${ }^{(5)}$

Beetroot (Beta vulgaris) is high in immune-boosting vitamins \& minerals; it is also good for the bones, liver \& kidneys, and it reduces risk of heart disease by modulating the blood pressure. (6) The petals of Rose (Rosa) are beneficial to the skin \& cure dry and patchy skin; they are also used in health tonics. Carrot (Daucus carota) is a rich source of vitamins A, C, K, B-8, as well as pantothenic acid \& folate, and ions like potassium, iron, copper, and manganese. It is also a good source of antioxidants. (10) Marigold (Tagetes) is famous for its use in herbal ointments. It is a rich source of flavonoids and anti-oxidants. Pomegranate peels (Punica granatum) are effective detoxifying agents. It acts as a combat tool against heart diseases, and in prevention of skin cancer. It is also known to cure coughs \& sore throats. ${ }^{(9)}$ Henna (Lawsonia inermis) plays essential role in detoxification, skin protection, boosting hair health, etc. Spinach (Spinacia oleracea) is high in protein, zinc, fibre, vitamins, etc. Its health benefits include skin care, eyesight, regulated blood pressure etc. It is a good source of water-soluble vitamins like thiamine, riboflavin, niacin \& ascorbic acid, and contains enough amount of vitamin $\mathrm{C}$ to prevent and cure scurvy. (7) Peppermint (Mentha piperita) has 
significant anti-microbial and anti-viral ability, strong anti-oxidant and anti-tumor actions, and also exhibits anti-allergenic potential to a certain extent $^{(8)}$.

Bioactive compounds in plants are instrumental for various beneficial activities of the plant. (11) Phytochemical assays are often considered to be the first step for characterization of a plant depending on the presence of the various metabolites. A positive response of the plant sample for the presence of Terpenoids, Flavonoids \& Glycosides indicates it potential use for defence against microorganisms, since these metabolites impart the ability to inhibit the growth of organisms. (12)(13)(15) Phenolic compounds possess properties like antiaging, anti-cancer, anti-inflammatory, antiatherosclerosis, and provides cardiovascular protection. (14) Coumarins have bacteriostatic and anti-tumor activity, they mediate inflammatory responses, and act as anti-coagulants. (16) Quinones provide protection against reactive oxygen species (ROS) \& have a diverse role in therapeutics, including anti-cancer agents and anti-aging and arteriosclerosis. (17) Free radicals are highly reactive, and are capable of damaging cells. They are associated with inflammatory diseases like rheumatoid arthritis and respiratory disorders. Various studies have demonstrated the adverse effects of anti-oxidant supplements, such as high risk of cancer, increased mortality, etc. Several nutrients in food and/or plants, like vitamin $\mathrm{C}$ and $\mathrm{E}$, beta-carotene, phenols, flavonoids, etc. are commonly studied dietary anti-oxidants. ${ }^{(18)}$

Anti-microbial agents are essentially important in reducing the burden of infectious diseases. Alternative anti-microbials are needed, and this has led to a re-evaluation of ancient remedies of plants and plant-based products. The use of plant extracts and its phytochemicals can be of great importance in treatment of microbial infections ${ }^{(19)}$. With regards to the increased scope of natural dyes, its study, production, and usage, the present research is designed for the extraction of dyes from natural plant sources, studying its essential characteristics and carrying out applications of the same.

\section{Materials and Methods}

Selection \& collection of plant materials: Eight different plant sources were chosen for extraction of dyes of 4 different colors. Beetroot \& Rose for Red, Pomegranate peels \& Henna for Brown, Spinach \& Peppermint for Green, Carrot \& Marigold for Orange.

Extraction of natural dyes: Dyes were extracted using both water \& organic solvents. Water-based dyes were prepared by blanching, grinding \& filtering the plant material using distilled water. (4)(5) They were further concentrated using lyophilization. Organic solvents were used for extraction of the color imparting phytochemical. Methanol was used for beetroot, rose, marigold, and henna. (20)(21) Ethanol was used for pomegranate peels, while petroleum ether was used for carrot. Spinach \& peppermint were extracted using acetone as solvent. (22)(23)(24)

Qualitative phytochemical analysis: The waterbased dyes were used for phytochemical screening. Phytochemicals like proteins, glycosides, terpenoids, quinones, coumarin \& saponins were analysed. (11) Phenols, tannins, carbohydrates, and flavonoids were also analysed. (25)(20)

Antimicrobial ability: The ability of the dyes to inhibit bacterial growth was tested using the Agar dilution method. $1 \mathrm{~g}$ of concentrated dye mixed with $2 \mathrm{ml} \mathrm{20 \%}$ DMSO was incorporated into molten nutrient agar butts which were poured onto petriplates. 6 common pathogenic organisms, namely, E. coli, S. typhi, P. aeroginosa, S. aureus, S. faecalis and $C$. diphtheriae, were spot inoculated on the plates and incubated to check for growth. (26)(27)

Anti-oxidant activity: The ability of the dyes to scavenge free oxygen radicals was determined using the FRAP (Ferric Reducing Ability of Plasma) assay. (21)

\section{Applications of the dyes:}

Use as Food coloring agents: Noodles were colored by boiling them in water containing the dye for 5-10 minutes; while, doughs of white flour were prepared using dye mixed with water.

Cosmetic production (Lip-balm): Natural ingredients like aloe gel, almond oil, beeswax, rice powder \& cornstarch were mixed well. 5-6 drops of the dye was added and mixed until a good consistency was obtained. The prepared lip-balms were then stored at $4^{\circ} \mathrm{C}$ and $\mathrm{RT}$.

\section{Results}

The extracted dyes showed a good color imparting ability and were of the desirable consistency.

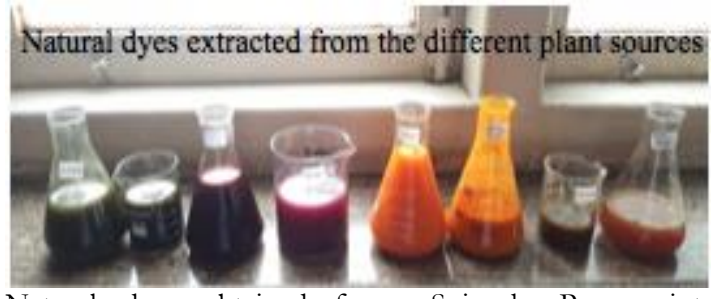

Natural dyes obtained from- Spinach, Peppermint, Beetroot, Rose, Carrot, Marigold, Henna, Pomegranate peels. ( $\mathrm{L}$ to $\mathrm{R}$ )

Qualitative

phytochemical analysis: Phytochemical analysis revealed the presence of important metabolites like Proteins, Carbohydrates, 
Phenols, Tannins, Terpenoids, and Glycosides. These are major metabolites present which are responsible for several additive properties of the plants.

Fig. 1: Tabular representation of phytochemical analysis of the dyes.

\begin{tabular}{|c|c|c|c|c|c|c|c|c|}
\hline $\begin{array}{l}\text { Dyes } \\
\text { Compound }\end{array}$ & Beetroot & Rose & \begin{tabular}{|l|} 
Pom \\
peel
\end{tabular} & Henna & Spinach & $\begin{array}{c}\text { Pepper } \\
\text { mint }\end{array}$ & Carrot & Marigold \\
\hline Proteins & + & + & + & + & + & + & + & - \\
\hline Carbohydrates & + & + & + & + & + & + & + & + \\
\hline Flavonoids & - & + & + & $\cdot$ & + & + & + & + \\
\hline Glycosides & + & + & + & + & + & + & - & + \\
\hline Terpenoids & + & + & + & + & - & + & + & - \\
\hline Saponins & + & + & + & + & + & + & - & + \\
\hline Phenols & + & + & + & + & + & + & + & + \\
\hline Tannins & + & + & + & + & + & + & - & + \\
\hline Quinones & + & + & + & + & - & + & - & $\cdot$ \\
\hline Coumarin & + & + & + & $\cdot$ & $\cdot$ & $\cdot$ & - & + \\
\hline
\end{tabular}

Antimicrobial ability: The dyes were examined for their antimicrobial ability by the Agar Dilution method. Organisms like $S$. aureus and $P$. aeroginosa, were inhibited by all the dyes. While, S. typhi, E. coli, $C$. diphtheriae and $S$. faecalis were inhibited by most of the dyes. The following is a tabular representation of the antimicrobial ability of the dyes as observed, upon comparing with the controls.

Fig. 2: Tabular representation of the antimicrobial ability of the dyes

\begin{tabular}{|c|c|c|c|c|c|c|}
\hline $\begin{array}{l}\text { Organism } \\
\text { Dyes }\end{array}$ & E. coli & S. typhi & P. aeroginosa & S. aureus & S. faecalis & C. diphtheriae \\
\hline Beetroot & 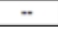 & - & - & - & 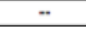 & $*$ \\
\hline Rose & + & -. & - & - & + & .* \\
\hline Pom peel & 4 & - & - & - & + & -. \\
\hline Henna & $\overline{-}$ & 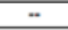 & - & 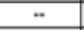 & 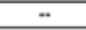 & 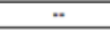 \\
\hline Spinach & + & $*$ & - & $*$ & $\cdot$ & $\cdot$ \\
\hline Peppermint & - & - & - & - & - & 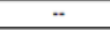 \\
\hline Carrot & - & + & + & - & + & + \\
\hline Marigold & 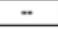 & 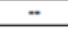 & - & $\ddot{*}$ & $\ddot{*}$ & + \\
\hline Media Control & 4 & + & + & + & 4 & + \\
\hline DYSO Control & + & + & + & + & + & + \\
\hline
\end{tabular}

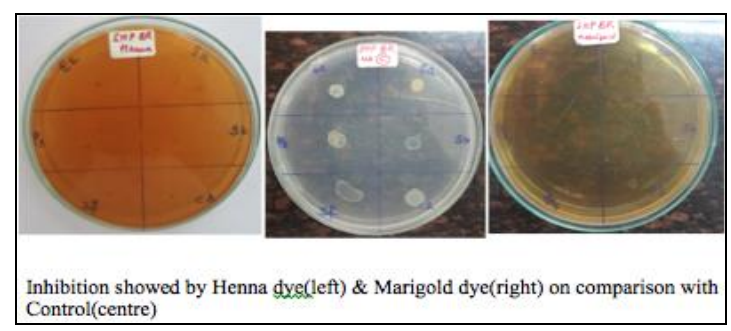

Anti-oxidant activity: The anti-oxidant activity of the dyes was tested using FRAP assay \& ascorbic acid as standard. Highest anti-oxidant activity was shown by dye obtained from beetroot, followed by henna, rose and carrot dye. Other dyes also showed good anti-oxidant activity.

\section{Applications of the dyes:}

Use as Food coloring agents: The noodles \& whilte flour dough that were prepared using the dyes retained a good color, and no considerable change in taste was imparted to the food products.
Fig. 3: FRAP values of the dyes in descending order of the anti-oxidant activity. (Higher the FRAP value, greater the anti-oxidant activity)

\begin{tabular}{|c|c|}
\hline Dyes & FRAP value \\
\hline Beetroot & 2.00 \\
\hline Henna & 1.99 \\
\hline Rose & 1.98 \\
\hline Carrot & 1.97 \\
\hline Pom peel & 1.94 \\
\hline Spinach & 1.86 \\
\hline Marigold & 0.90 \\
\hline Peppermint & 0.62 \\
\hline
\end{tabular}

Fig. 4: Graphical representation of FRAP values of ascorbic acid (standard)
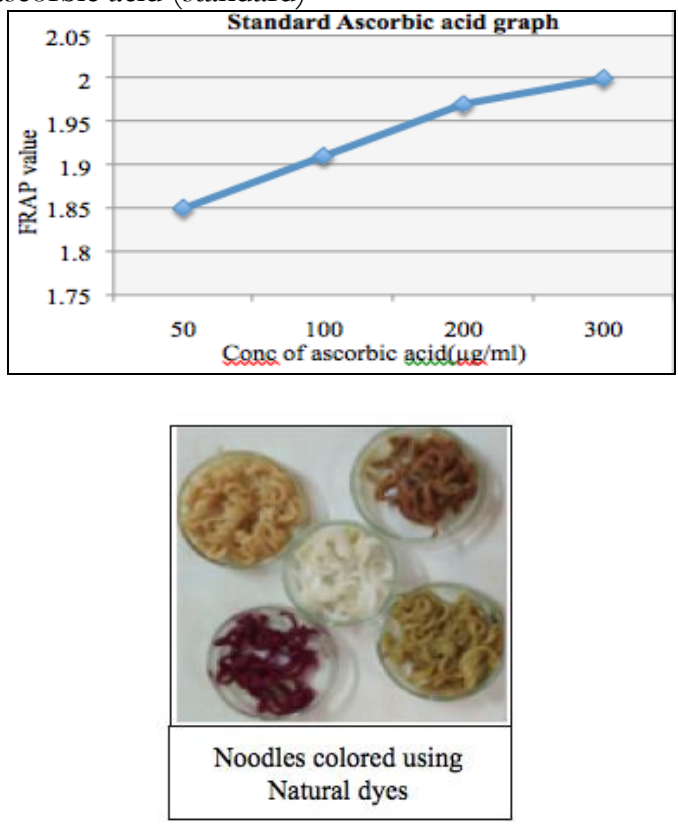

Cosmetic production (Lip-balm): The prepared lip-balms were found to be stable upon storage at RT \& $4^{\circ} \mathrm{C}$ for upto 4 weeks. They had a good consistency and gave a vibrant color. Also, since the dyes were prepared using all natural ingredients, they gave a refreshing and soothing feel to the skin.

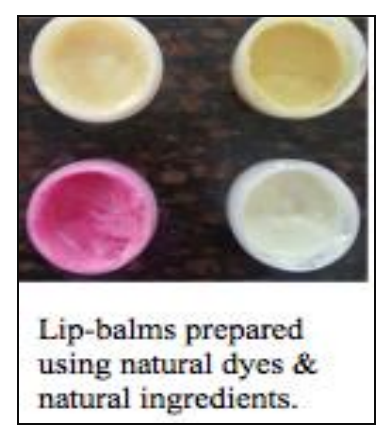

\section{Discussion}

The dyes extracted from natural sources are rich sources of important bioactive compounds. Plants like Henna, Beetroot, Carrot, Spinach, etc are known to have therapeutic properties. The extracted dyes showed prominent and lucid colors. 
Upon testing for presence of various phytochemicals, the dyes showed presence of important metabolites like proteins, tannins, carbohydrates, phenols, glycosides and many more. These phytochemicals are known to have therapeutic properties, like protecting the cellular systems from oxidative damage, modulating immune system, preventing cancer and lowering the risk of chronic diseases like cardiovascular diseases, etc. The antimicrobial ability of the dyes was tested using the agar dilution method \& the dyes showed inhibition to common pathogenic organisms like Staphylococcus aureus, which is a common skin infection causing bacterium, and, Pseudomonas aeroginosa, which is causes various nosocomial infections. Organisms like Streptococcus faecalis, Salmonella typhi, Escherichia coli, and Corynebacterium diphtheriae, that are the causatives of various afflictions were also inhibited by most of the dyes. Being extracted from natural plant sources, the dyes were found to be rich in anti-oxidants. The highest anti-oxidant level found spectrophotometrically was that of the Red beetroot dye (FRAP value $=2.00)$. The other dyes obtained were also found to have good anti-oxidant activity. The food products colored using the dyes showed no change in taste \& retained a vibrant color. Also, the cosmetic (lipbalms) prepared using the dyes were found to be stable upon storage \& gave a refreshing and soothing feel to the skin.

Natural dyes are on the rise ever since the harmful effects of artificial dyes have begun to uncover. Presence of important metabolites and high antioxidant activity are an indication of the beneficial purposes these dyes will serve in food industries. The ability to inhibit commonly pathogenic organisms is proof of the medicinal \& therapeutic properties of these natural dyes. Natural dyes can, thus, replace artificial dyes in the near future.

\section{Acknowledgement}

The authors are thankful to Jigyaasa - Science Honors Program, K.C College, under which the study was carried out. We are also thankful to the University of Mumbai for partly funding the research project.

\section{References}

1. Abrahart, Edward Noah, and J.B. Stothers. "Dye" Encyclopadia Britannica, 18.03 (2018). Online.

2. Laurel Curran. "Food Dyes Linked to Cancer, ADHD, Allergies." Food Safety News. (2010). Online.

3. "Natural vs. Synthetic dyes: which is better?" Key Color, (2015)

4. Saxena, Sujata, and A.S.M Raja. "Natural Dyes: Sources, Chemistry, Application and Sustainability Issues." Roadmap to Sustainable Textiles and Clothing, Springer 1 (2014): 37-80. Print.
5. Samanta, Ashis Kumar, and Adwaita Konar. "Dyeing of Textiles with Natural Dyes." Natural Dyes (2011): 29-56. Print.

6. Clifford, Tom and Glyn Howatson, et al., "The Potential Benefits of Red Beetroot Supplementation in Health and Disease." Nutrients 7.4 (2015): 28012822. Online.

7. Hanif, Rumeza, and Zafar Iqbal, et al., "Use of vegetables as nutritional food: role in human health." Journal of Agricultural and Biological Science 1.1 (2006): 18-22. Online.

8. McKay, Diane, and Jeffrey Blumberg. "A review of the bioactivity and potential health benefits of peppermint tea (Mentha piperita L.)" Phytotherapy Research 20.8 (2006): 619-633. Online.

9. Viuda-Martos, Manuel, and Javier Fernández-López, et al., "Pomegranate and its Many Functional Components as Related to Human Health: A Review." Comprehensive Reviews in Food Science and Food Safety 9.6 (2010): 635-654. Online.

10. Marina Heinonen. "Carotenoids and provitamin A activity of carrot (Daucus carota L.) cultivars." Journal of Agriculture and Food Chemistry 38.3 (1990): 609-612. Print.

11. Shah, Shreshtha, and Anushi Jain, et al., "Study of total phenol, flavonoid contents and phytochemical screening of methanolic crude extracts of two weed plants." Annals of Plant Sciences 6.6 (2017): 1645-1648. Online.

12. Havsteen BH. "The biochemistry and medical significance of the flavonoids." Pharmacology and Therapeutics 96.2-3 (2002): 67-202. Online.

13. Fu G, Pang $\mathrm{H}$, et al., "Naturally occurring phenylethanoid glycosides: potential leads for new therapeutics." Current Medicinal Chemistry, 15.25 (2008): 2592-613. Online.

14. Yadav, RNS, and Munin Agarwala. "Phytochemical analysis of some medicinal plants." Journal of Phytology 3.12 (2011): 10-14. Online.

15. Ghosh, Pranab, and Amitava Mandal, et al., "Triterpenoids from Psidium guajava with biocidal activity." Indian Journal of Pharmaceutical Sciences 72 (2010): 504-507. Online.

16. Jain, P.K, and Himanshu Joshi. "Coumarin: Chemical and Pharmacological Profile." Journal of Applied Pharmaceutical Science 2.6 (2012): 236-240. Online.

17. Madeo, Jennifer, and Adeel Zubair, et al., "A review on the role of quinones in renal disorders." SpringerPlus 2.1 (2013): 139. Online.

18. Satyanarayana, Uppala, and Amar Nagesh Kumar, et al., "Antioxidant supplementation for health - a boon or a bane?" Journal of Dr.NTR University of Health Sciences 3.4 (2014): 221-30. Online. 
19. Nascimento, Gislene G. F., and Juliana Locatelli, et al., "Antibacterial activity of plant extracts and phytochemicals on antibiotic-resistant bacteria." Brazilian Journal of Microbiology 31.4 (2000): 247-256. Print.

20. Tiwari, Prashant, and Bimlesh Kumar, et al., "Phytochemical screening and Extraction: A Review." Internationale Pharmacentica Sciencia 1.1 (2011): 98-106. Online.

21. Maruthamuthu, Vijayalakshmi, and Kandasamy Ruckmani. "Ferric reducing anti-oxidant power assay in plant extract." Bangladesh Journal of Pharmacology 11 (2016): 570-572. Online.

22. Khan, M. Ali, and M. Khan, et al., "Extraction of natural dyes from myrobalan, gallnut and pomegranate, and their application on wool." Colourage (2005): 53-60. Print.

23. Straumite, Evita, and Z. Kruma, et al., "Pigments in mint leaves and stems." Agronomy Research 13.4 (2015): 1104-1111. Print.

24. Hecker, Susan. "Extraction of B-carotene from orange peel and carrot waste for cotton dyeing." The Swedish School of Textiles (2014). Print.
25. Chandrashekar, R, and S.N Rao. "Phytochemical analysis of ethanolic extract of leaves of Leucas indica (eelli)." International Journal Of Pharma And Bio Sciences 4.1 (2013): 33-38. Online.

26. Wiegand, Irith, and Kai Hilpert, et al., "Agar and broth dilution methods to determine the minimal inhibitory concentration (MIC) of antimicrobial substances." Nature protocols 3.2 (2008): 163-175. Online.

27. Nostro, A., and M.P Germanò, et al., "Extraction methods and bioautography for evaluation of medicinal plant antimicrobial activity." Letters in Applied Microbiology 30 (2000): 379-384. Online.

\section{Cite this article as:}

Bushra Khan, Ramya Sindhyan, Anushi Divan, Sejal Rathod. Extraction, characterization \& applications of natural dyes. Annals of Plant Sciences 7.11 (2018) pp. 2463-2467.

do http://dx.doi.org/10.21746/aps.2018.7.11.4 